Rabaska

Revue d'ethnologie de l'Amérique française

\title{
Politique culturelle 2019 : instaurer un leadership de concertation
}

\section{Gérald Grandmont}

Volume 17, 2019

URI : https://id.erudit.org/iderudit/1066020ar

DOI : https://doi.org/10.7202/1066020ar

Aller au sommaire du numéro

Éditeur(s)

Société québécoise d'ethnologie

ISSN

1703-7433 (imprimé)

1916-7350 (numérique)

Découvrir la revue

Citer ce document

Grandmont, G. (2019). Politique culturelle 2019 : instaurer un leadership de concertation. Rabaska, 17, 259-272. https://doi.org/10.7202/1066020ar d'utilisation que vous pouvez consulter en ligne.

https://apropos.erudit.org/fr/usagers/politique-dutilisation/ 


\title{
Opinion
}

\section{Politique culturelle 2019 : instaurer un leadership de concertation}

\author{
GÉRALD GRANDMONT \\ Professeur associé HÉc Montréal \\ et Université du Québec à Trois-Rivières
}

\section{Introduction}

Exprimer une opinion sur la nouvelle politique culturelle pour une revue qui porte le nom de Rabaska oblige à des considérations particulières. Pour ainsi dire, cela donne le ton de l'exercice à venir. Ce nom de « rabaska » est d'origine algonquienne ; c'est le nom d'un canot de grande taille qui favorisa la pénétration de la culture francophone sur le continent. Par analogie, il en va de même de la culture portée aujourd'hui par le verbe, l'image et le numérique qui servent de véhicules de rayonnement.

La politique culturelle en cours datait de 1992. Plus de 27 ans sans qu'elle ne soit mise à jour est une longue période, vu la rapidité des changements survenus au cours de ce quart de siècle. Pour mesurer la portée de ces changements, rappelons-nous qu'Internet en était à ses premiers balbutiements à cette date et que la majorité de la population ignorait ce dont il s'agissait. Certes, certains programmes furent revus en cours de route et d'autres mis en place, de nouvelles législations virent le jour, mais fondamentalement le registre fut sensiblement le même.

Ce qu'il y a encore de singulier de cette nouvelle politique culturelle, c'est que l'accouchement du présent projet de politique fut le fruit d'une longue démarche portée par trois ministres distincts du gouvernement Couillard, soit Hélène David qui l'a lancée, Luc Fortin qui l'a poursuivie et Marie Montpetit qui l'a rendue à terme en juillet 2018. Une situation inusitée qui voit un tel projet porté par trois personnes différentes et qui, de surcroît, sera implanté par une quatrième ministre, Nathalie Roy, membre d'un autre gouvernement, celui de François Legault en 2019. 


\section{De grandes orientations}

Cette nouvelle politique expose un projet avec une remarquable largeur de vue, des sensibilités nouvelles et des orientations qui dépassent le strict univers des arts, des lettres et du patrimoine pour embrasser des dimensions sociales, économiques, internationales et de la communication tout en optant pour un angle d'attaque opérationnel qui se concrétise dans un amalgame de 34 mesures dominantes. Voilà le signe d'un sain rafraîchissement pour l'espace que doit occuper la culture dans une société.

D’entrée de jeu, cette politique expose ses assises sociales par « la valorisation du caractère essentiel de la culture et de la langue française dans la vie des gens et des collectivités, entre autres par la reconnaissance de leur lien indissociable avec l'éducation ${ }^{1} \gg$. Un premier signal que le lien avec les citoyens et les citoyennes a son importance.

Elle enchaîne avec le renouvellement de « son soutien aux artistes professionnels et aux créateurs ainsi qu'aux travailleurs, industries et organismes culturels. ${ }^{2} \gg$ On observe ici le maintien d'une forme de surdétermination du monde des arts et des lettres et des industries culturelles dans les grands choix, ce qui est tout à fait légitime. La priorité du soutien à la création, la diversité des formes de création de même que la recherche du rayonnement des arts et de la culture sont à la fois culturellement et socialement marqués, tout comme l'amélioration souhaitée de la condition socioéconomique des artistes ainsi que la conjoncture du numérique et des changements qui appellent des programmes adaptés.

La troisième orientation introduit la notion de territoire et elle « vise à ce que l'engagement des collectivités, la présence d'établissements et d'organismes culturels ainsi que la conservation du patrimoine contribuent à la qualité des milieux de vie ${ }^{3} »$. C'est la manière par laquelle cette politique choisit de graver l'apport de la culture, des arts et surtout du patrimoine dans l'aménagement du territoire en misant notamment sur une plus grande implication des municipalités et une mobilisation des citoyens. Cette politique reconnaît encore des rôles spécifiques pour Montréal et Québec.

La quatrième se tourne vers l'apport de la culture à l'économie et au développement du Québec en cherchant à favoriser un «plein essor d'une société créative, innovante et prospère ${ }^{4} »$. Internet est un outil de mondialisation et de dialogue, et le numérique est le langage dématérialisé et mathématique qui l'alimente. Trois enjeux surgissent au premier regard sous ce rapport,

1. Partout la culture, politique culturelle, Gouvernement du Québec, 12 juin 2018, [62p.] ; cf. mcc.gouv.qc.ca/fileadmin/documents/Politique_culturelle/Partoutlaculture_Polculturelle_Web.pdf, p. 10.

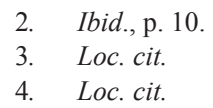


celui du développement d'un entreprenariat, celui d'une fiscalité adaptée au contexte numérique, et celui de la « découvrabilité ${ }^{5}$ » des œuvres et des produits québécois sur les marchés internationaux.

Enfin, cette politique accorde une attention particulière aux autochtones et aux Inuits en inscrivant un « Plan d'action gouvernemental pour le développement social et culturel des Premières Nations et des Inuits 2017$2022^{6}$ ». C'est là une première dans une politique culturelle au Québec. Cette ouverture arrive d'autant à point nommé que le Conseil des arts et des lettres du Québec s'emploie depuis quelques temps à mettre en place de nouveaux programmes pour les autochtones, que ce soit à l'égard de la valorisation des cultures ancestrales ou de la formation et de l'intégration d'artistes autochtones dans les milieux professionnels.

Cette politique a encore l'avantage d'être investie d'un Plan d'action quinquennal, lui-même assorti de l'annonce d'un engagement financier majeur. Alors, que devons-nous retenir de cette politique multiforme ? Quelles en sont les nouveautés, les exigences, les contraintes et les limites?

\section{Des avenues nouvelles}

Cette politique ouvre la voie à plusieurs avenues nouvelles qui élargissent et enrichissent le spectre de l'action culturelle de l'État. J'en mentionne un certain nombre.

\section{La langue}

Trop longtemps cette responsabilité collective majeure fut dissociée de la culture, étant confiée à des ministres différents. La politique propose de voir « le gouvernement encourager les acteurs publics [...] à apporter leur contribution à la promotion du français. Il souhaite ainsi créer une synergie entre la culture et la langue française, par exemple en se dotant de stratégies de valorisation ou en améliorant le dialogue entre les différentes communautés linguistiques du Québec ${ }^{7} \gg$. Cela suffira-t-il ? Si l'attraction du français a connu un accroissement grâce à la francisation des immigrants par l'école, elle montre désormais plusieurs signes de fragilité sur l'accueil en français dans les commerces à Montréal, sur la langue de travail où le français est en recul, et de surcroît chez les jeunes francophones eux-mêmes qui affichent une indifférence face à un service dans une autre langue ${ }^{8}$. Considérant son

5. Découvrabilité : ce potentiel pour qu'un produit ou une œuvre capte l'attention des internautes pour stimuler leur intérêt à en repérer d'autres. Une approche qui modifie considérablement les stratégies de visibilité.

6. $\quad$ Partout la culture, politique culturelle, op. cit., p. 50.

7. Ibid., p. 18.

8. Office québécois de la langue française, extraits du Rapport sur l'évolution de la situation linguistique au Québec, communiqué de presse, 12 avril 2019. 
faible poids démographique en Amérique du Nord, la langue française aura ici toujours besoin de balises de protection autant que de mesures de valorisation. Le respect d'une langue commune dans la sphère publique implique que tous les ministères soient concernés et que les citoyens soient fiers de cette langue française.

\section{La création, la production et la diffusion culturelle}

Donner accès à un continuum de formation professionnelle et de perfectionnement, favoriser l'emploi, la rémunération et la protection sociale des artistes, favoriser l'équité entre les hommes et les femmes sont des vecteurs connus. Ainsi, les artistes du cirque disposeront désormais des mêmes conditions d'entraînement que les danseurs dans un cadre couvert par la Commission des normes, de l'équité, de la santé et de la sécurité du travail (CNÉssT). À cet égard, il s'agit moins d'inventer des nouvelles solutions que d'accroître les aides gouvernementales à la création et à la production qui sont des mesures fort attendues.

Deux vecteurs méritent cependant une attention particulière : la diversité culturelle et l'éclatement des disciplines induite par le numérique. Il apparaît que les milieux culturels sont davantage mûrs pour s'ouvrir concrètement à la prise en compte de la diversité culturelle aussi bien dans les courants de création que dans le choix des artistes à l'interprétation. Pour supporter cette ouverture, le Conseil des arts et des lettres du Québec (CALQ) et la Société de développement des entreprises culturelles (SODEC) doivent adopter une posture rapide d'adaptation des programmes et offrir des incitatifs à la réalisation des transformations.

Sous l'impulsion du numérique, l'éclatement disciplinaire et la pratique professionnelle multiforme posent de nouvelles questions à la reconnaissance professionnelle de qui est un metteur en scène, un artiste, un réalisateur, un technicien, rôles joués de plus en plus souvent par la même personne. Il faut alors adapter rapidement les dispositions des lois sur le statut de l'artiste. Ces lois se présentent comme un complément au code du travail et elles devront prendre en compte ces nouvelles définitions de l'artiste. De même, la création numérique trouve difficilement accès à un financement public avec des statuts d'entreprises hybrides et une multidisciplinarité omniprésente qui s'écarte du seul registre du jeu vidéo, bien que ce soit sans doute celui-ci qui a ouvert la voie à de multiples « déclinaisons » nouvelles. Ici encore, les programmes de la SODEC et du CALQ seront sollicités pour harmoniser des plateformes souples et communes de soutien. Il en va de même des circuits de formation qui peinent à suivre le rythme accéléré du mouvement. «Il faut que nos institutions et nos bailleurs de fonds s'ouvrent à ces nouveaux modèles de 
production, qu'ils soient ouverts à l'interdisciplinarité. Les œuvres pour les dômes, ça reste de la fiction ou du documentaire, mais c'est juste que l'écran n'est pas plat. Il faut régler ça le plus rapidement possible », explique Jenny Thibault, présidente de Xn Québec, l'Association des producteurs d'expériences numériques ${ }^{9}$.

\section{Le patrimoine}

La Loi sur le patrimoine culturel n'a que sept ans. On avait espéré qu'elle susciterait rapidement de nouveaux réseaux et de nouvelles sensibilités de protection et de mise en valeur. Celles-ci tardent à s'exprimer en certains milieux parce qu'elles ne trouvent leurs points d'appui que dans le temps long et après des efforts de mobilisation. À l'heure où l'incendie de la cathédrale Notre-Dame de Paris a suscité des émotions et des engagements de partout dans le monde, cela nous rappelle des distinctions de fond : « Dans le patrimoine, on ne ravale pas, on ne reconstruit pas, on restaure selon les principes de la Charte de Venise... ${ }^{10} \gg$ En même temps, force est de constater que l'implication des milieux municipaux passe sans doute par une perception de la valeur sociale et communautaire du patrimoine, ce qui est en proximité avec les citoyens. C'est une observation que l'on peut retenir du recyclage des églises désaffectées de fonctions du culte. C'est également, avec de telles notions sociales et communautaires, qu'on peut mieux inscrire le patrimoine comme un actif de l'aménagement du territoire. En outre, avec un recours à des crédits d'impôt conjoints mis à la disposition de partenaires privés et une majoration du soutien public, on arriverait sans doute à créer un mouvement favorable à la valorisation du patrimoine dans le recyclage de bâtiments auprès des municipalités et des partenaires privés. S'ajoute désormais à ces dimensions celle d'une fort opportune stratégie de l'architecture, laquelle trouve également son point de chute dans l'aménagement du territoire.

De même, le gouvernement doit se montrer exemplaire à l'égard du patrimoine. Il ne peut exiger des citoyens ce qu'il n'exige pas de lui-même. Prenons le cas du patrimoine public appartenant à d'autres ministères tels que les universités, les bâtiments hospitaliers, les ouvrages d'ingénierie, les équipements agricoles, etc. Ces ministères disposent dans leurs budgets d'immobilisation d'une mesure de maintien des actifs qui représente un pourcentage de la valeur de ces équipements pour les maintenir en état. Le ministère de la Culture et des communications profite d'une majoration de ce pourcentage afin de protéger le caractère patrimonial de certains biens immobiliers; il faut

9. Philippe Papineau, « La Créativité numérique en plein boom », Le Devoir, 2 février 2019.

10. Gil Mihaely, « Le patrimoine gêne, il est perçu comme contraire à la modernité », entretien avec Maryvonne de Saint-Pulgent, $1^{\text {er }}$ mai 2019 ; $c f$. www.causeur.fr/saint-pulgent-notre-dame-patrimoine-161039. 
obtenir une majoration comparable pour les autres ministères gestionnaires de bâtiments publics afin d'y protéger leur richesse patrimoniale.

Si la dimension du patrimoine doit aujourd'hui s'arcbouter dans l'action interministérielle, elle doit également s'inscrire dans l'aménagement du territoire. Elle devient alors une responsabilité partagée avec les municipalités et les MrC. Cette porte fut ouverte par la Loi sur le patrimoine culturel de 2012. À ce moment, une vaste entreprise de sensibilisation des élus et des administrateurs municipaux avait eu cours. Cependant, la réalité des derniers mois a rattrapé les bonnes intentions par les nombreux ratés de certaines villes et par un manque de volonté, d'expertise et d'encadrement patrimonial dans la gestion de plusieurs villes qui fait que des mesures de précaution font largement défaut. D'autant que les décisions municipales n'ont très souvent que la durée du mandat d'un conseil municipal. Il en résulte des tempêtes patrimoniales démobilisatrices qui viennent brouiller les efforts consentis.

Il y a ici une conviction à introduire auprès des élus et des gestionnaires municipaux de l'importance de cette dimension du patrimoine dans les plans d'urbanisme et d'aménagement du territoire. En réalité, ces plans évoluent à cloche-pied si une telle dimension en est absente. La mobilisation locale des élus et des personnes passe-t-elle par une conception désormais sociale et communautaire du patrimoine tout autant que par la définition des patrimoines à valeur historique, architecturale, archéologique, paysagère, de commémoration, immatérielle et autres ? Faut-il appuyer les décisions municipales en faveur du patrimoine, par des incitatifs fiscaux qui pourraient compenser les pertes d'une nouvelle fiscalité foncière et greffer à la décision de citer un bâtiment des apports juridiques et réglementaires pour protéger une telle décision $^{11}$ ? L'ingénieur Yves Lacourcière a publié un ouvrage le 11 juin 2018, soit la veille du lancement de la politique culturelle où il explique notamment que les États-Unis ont aligné le code d'impôt fédéral en 1976 « avec la politique nationale de préservation historique pour encourager les investissements du secteur privé volontaire dans la préservation des bâtiments historiques... et que ce programme de partenariat avec le secteur privé est particulièrement créateur d'emplois... ${ }^{12}$ » Voilà un exemple probant qui montre comment la fiscalité peut contribuer au développement de métiers du patrimoine en leur offrant un marché significatif.

Alors, cette avenue d'insertion du patrimoine dans le creuset de l'aménagement du territoire implique un accompagnement des acteurs, un accès

11. Lire à ce sujet l'article de Jean-François Nadeau du quotidien Le Devoir du 4 mai 2019 intitulé « La valeur des taxes foncières dicte-t-elle le sens des décisions prises par les municipalités? Deuxième volet : le patrimoine. »

12. Yves Lacourcière, Rapport d'enquête. Accusé de non-assistance à patrimoine en danger ... la mort annoncée de nos patrimoines du bâti, Contrad international, éditeur, p. 286-287. 
aux expertises utiles et cela commande une participation par le gouvernement aux financements de pareils services. Faudrait-il encore ici ajouter aux côtés des ententes de développement culturel une entente spécifique prenant en compte la réalité culturelle et patrimoniale dans le cadre de l'aménagement du territoire avec les municipalités et les MRC ? Ces dernières disposent dans un certain nombre de cas de professionnels que de nombreuses municipalités n'ont pas et elles pourraient sans doute assumer la responsabilité de tenir à jour un inventaire du patrimoine bâti à préserver.

Bref, l'état du patrimoine partout sur le territoire commande des virages significatifs : pouvoirs réglementaires, soutiens financiers directs, crédits d'impôt, maintien des actifs publics majorés et services d'inventaire, de formation et d'expertise. Un plan d'ensemble.

\section{Communications et médias}

Les médias, dans un rapport inversé, sont désormais placés dans la situation de s'ériger en contrepoids aux médias sociaux qui sont un réservoir d'opinions exprimées parfois sans nuances et qui peuvent comporter de fausses vérités (fake news) sans aucun recul. Historiquement, les médias ont contribué au maintien d'une cohésion sociale indispensable à la vie en société. C'est encore vrai aujourd'hui et l'implantation dès 2018 de crédits d'impôt sur la masse salariale du personnel producteur d'informations est opportune, mais celle-ci ne suffira pas à compenser le transfert des revenus publicitaires en faveur des $\mathrm{GAFA}^{13}$ de ce monde. Il faut encore aller plus loin et accepter de soutenir par des moyens financiers publics leur production et leur maintien selon des règles claires et explicites qui pourraient notamment s'apparenter à des mesures d'appariement de contributions privées des citoyens et des corporations. Et cela passe également par une concertation avec le gouvernement canadien.

\section{Le numérique}

La respiration de nos sociétés contemporaines trouve son principal volume d'oxygène dans Internet. Les tribunes d'échanges, de discussions, de débats, voire de confrontations ou d'agressions prennent place dans les médias sociaux, y compris la manipulation politique des démocraties, lesquels médias ont développé des modèles d'affaires fondés exclusivement sur la publicité. Ces médias drainent consommateurs et citoyens dans des sphères qui échappent pratiquement à toutes règles.

La politique prévoit des mesures qui visent à permettre l'éclosion de l'entrepreneuriat culturel, à former et à réunir les compétences numériques et à développer de nouveaux marchés appuyés sur la découvrabilité des œuvres

13. L'acronyme GAFA désigne les quatre grandes entreprises d'Internet: Google, Apple, Facebook, Amazon. 
et à obtenir une équité fiscale pour tous afin de trouver une niche dans ces nouveaux réseaux.

Deux éléments retiennent ici l'attention : la mutualisation des connaissances $^{14}$ et l'équité fiscale. Il appartient aux milieux professionnels de se mettre au travail pour implanter des formules concrètes de mutualisation des connaissances entre les bailleurs de fonds publics, les entreprises nouvelles du numérique, les milieux artistiques et les universités. Par ailleurs, sur le plan de l'équité fiscale, considérant la très grande place occupée par les arts et les industries culturelles sur ces réseaux, peut-on légitimement imaginer qu'une part significative des revenus obtenus de cette nouvelle fiscalité initiée par le gouvernement québécois soit réinvestie directement dans la construction d'une force numérique plus ambitieuse?

A contrario, il est urgent que le gouvernement canadien lève la barrière du refus de cette nouvelle forme de fiscalité. Si la France peut prendre l'initiative de développer la sienne sans attendre un accord de l'Union européenne, rien ne justifie le gouvernement canadien d'attendre que le mouvement soit partagé sur le plan international.

Car, bien au-delà du strict avantage économique, de tels gestes forceraient peut-être le jeu d'une prise de conscience de l'utilité de règles éthiques, financières et respectueuses des personnes. Si ces médias en arrivent à dominer les gouvernements, la démocratisation s'en trouvera fragilisée et les outils de cohésion sociale indispensables à la vie collective en seront d'autant marginalisés.

\section{La dimension sociale des arts et du patrimoine}

Encourager les initiatives utilisant la culture comme outil d'intervention sociale, enrichir l'éventail des activités et des services adaptés aux besoins des personnes handicapées, immigrantes ou en situation de pauvreté qu'offrent les sociétés d'État en culture, encourager la tenue d'activités citoyennes collaboratives menant à la diffusion et à la mise en valeur de documents patrimoniaux québécois sur le Web, améliorer l'offre de sorties et d'activités culturelles dans le parcours éducatif, soutenir des projets culturels et d'engagement social pour les jeunes autochtones, offrir aux personnes immigrantes et aux personnes nouvellement arrivées au Québec un accompagnement en matière de culture dans les bibliothèques municipales sont autant d'avenues de la politique qui s'ouvrent sur des préoccupations sociales spécifiques, auxquelles s'ajoutent la modernisation du programme Mécénat placement culture et l'encouragement de la philanthropie culturelle par des mesures fiscales.

14. Il s'agit ici d'inviter les milieux culturels à mettre en commun connaissances, stratégies d'approches, partenariats dans la compréhension du modus operandi des réseaux Internet. 
Sous ce dernier rapport, il faut reconnaître que la culture a pris un retard certain sur la santé et l'éducation. Pourquoi ne pas songer à une bonification fiscale du don de citoyens ou de corporations à des organisations culturelles pendant dix ans afin de permettre à ce domaine de rattraper un tant soit peu son retard de philanthropie ? Le gouvernement y gagnerait à moyen terme. Certes, la règle fiscale prévoit que les mesures se doivent d'être communes à tous les domaines ; ce que nous évoquons ici, c'est de privilégier une disposition à géométrie variable sur une courte période.

\section{Un leadership de concertation}

Cette politique se positionne comme une politique gouvernementale. Cela implique que le ministère de la Culture et des communications se fasse chef d'orchestre de ce mouvement. La vision holistique de cette politique, les nouvelles dimensions prises en compte et les nombreux partenariats qu'elle sollicite appellent ainsi un puissant leadership de concertation pour construire des partenariats solides et pérennes. Cela exige de pouvoir compter sur plus d'un dispositif de soutien à une telle approche. La perspective du maintien, de l'enrichissement et de l'élargissement des concertations interministérielles (éducation, famille, immigration, relations internationales, environnement et développement durable, finances, affaires municipales, santé et agriculture) est clairement énoncée, tout comme un engagement plus grand des municipalités qui, elles, sont plus habiles que le gouvernement à soutenir des actions et des initiatives de proximité. Mais, qu'en est-il concrètement?

Sur le terrain de l'encadrement administratif et de la gouvernance de cette politique, on ne voit guère d'éléments pour en soutenir la réalisation, exception faite d'éventuels transferts aux sociétés d'État de la culture, d'une directive gouvernementale sur la préservation, la restauration et la réhabilitation des immeubles de l'État, ainsi que d'une nouvelle alliance culture, éducation et famille. Comment orchestrer une prégnance de la culture, des arts et du patrimoine qui puisse infléchir l'engagement concret des autres partenaires? Comment assurer un financement constant des initiatives chez ces mêmes partenaires ? Comment engager des études et des analyses continues du degré d'implantation de cette politique afin de la tenir constamment à jour?

Nous savons d'expérience, et les milieux culturels également, qu'il est fort difficile pour un ministère sectoriel d'agir sur l'ensemble de l'activité gouvernementale, le mandat du ministère de l'Environnement à l'égard du développement durable a pu, dans le passé, le laisser voir. Une telle dynamique passe par la recherche de partenariats gagnant-gagnant. Alors le ministère de la Culture et des communications devrait disposer de leviers financiers, tel un fonds d'initiatives culturelles (FIC) pour stimuler et apparier des actions 
d'autres ministères en relation avec les grands axes de cette politique culturelle. La France avait eu recours à une telle approche dans les années 1980. On pourrait encore créer des tables de concertation permanentes et interministérielles qui pilotent des initiatives croisées ; instaurer ainsi une gouvernance concertée de gestion de projets qui offrirait de meilleures chances d'ancrer la politique culturelle au cœur du développement.

Ce leadership de concertation devrait concerner la langue française, comme le propose cette politique. Pourquoi ne pas confier à l'Office québécois de la langue française (OQLF) un mandat-type de Commissaire à la langue française qui porterait un regard sur les actions entreprises par les ministères et les organisations publiques partenaires, et ferait rapport annuellement à 1'Assemblée nationale ${ }^{15}$ ?

De même, pour donner un ancrage encore plus grand à la concertation, il y aurait lieu d'ajouter à la Loi sur le patrimoine culturel une fonction de Commissaire au patrimoine qui aurait le mandat de fournir une évaluation des actions et des engagements ministériels et d'en faire rapport à l'Assemblée nationale.

\section{Une gouvernance de concertation}

Finalement, sous cette notion de leadership de concertation, je m'interroge sur le fait que le concept de la place de la culture dans le développement du Québec ne fasse pas partie du vocabulaire de cette politique, alors qu'elle évoque un large spectre de concertations interministérielles. Ce concept de culture et développement constituerait une image de marque qui donnerait à la culture une position centrale dans l'activité publique, comme a réussi à le faire le concept de développement durable $e^{16}$.

Car, si la politique a pour vertu d'être une politique gouvernementale comme expliqué dans les documents, pourquoi ne pas affirmer clairement qu'elle est cet actif du développement à enrichir auquel les autres partenaires ministériels et organisations parapubliques sont tenus de participer en se fixant des objectifs culturels dans la conception de leur contribution au développement collectif? N'est-ce pas la conception que s'en faisait le premier titulaire Georges-Émile Lapalme lorsqu'il écrivait dans son manifeste de 1959

15. Ces formules de " commissaire » sont désormais bien implantées dans le service public. Il existe un commissaire au développement durable, un commissaire à l'éthique, un commissaire au lobbyisme, un commissaire de la fonction publique, un commissaire à la lutte contre la corruption, un commissaire à la santé, lequel fut aboli un certain temps par l'ancien gouvernement et qui vient d'être rétabli par le gouvernement actuel.

16. À cet égard, l'implantation d'une telle démarche avait démarré par une loi-cadre ainsi qu'avec une historique réunion de tous les sous-ministres sous l'autorité du Secrétaire général du gouvernement, lequel avait été explicite dans ses propos quant à l'engagement que les sous-ministres étaient invités à prendre de piloter des actions de développement durable dans leur ministère respectif, et que la réalisation de ces actions ferait l'objet d'une évaluation annuelle. 
qu'il concevait le Québec comme un phénomène culturel ${ }^{17}$ ? Le Ministère ne saurait se contenter de tirer profit de conjonctures qui inciteraient d'autres partenaires publics à s'y associer.

Bref, un tel leadership de concertation assumé par le Ministère appelle non seulement des ressources humaines, mais également des dispositifs bilatéraux ou multilatéraux permanents, indispensables à son implantation : un Fonds d'initiatives culturelles, un commissaire à la langue française, un commissaire au patrimoine pour implanter une exemplarité de l'État ainsi qu'une majoration du pourcentage du maintien des actifs au profit de la protection du patrimoine immobilier. C'est de cette manière que le ministère de la Culture et des communications pourrait jouer le rôle de chef d'orchestre que la réalisation de cette politique attend de lui.

Le fait de passer sous silence un tel énoncé de la place de la culture dans le plan de développement du Québec a pour effet, pour les personnes qui connaissent les méandres de la vie politico-administrative du gouvernement, de constamment s'en tenir à une perception restreinte de la culture ce qui a souvent légitimé par le passé que tout cela ne doit guère peser qu'à peine plus d'un pourcent (1\%) dans les dépenses de l'administration publique.

L'Observatoire de la culture a déjà calculé avec beaucoup de rigueur que l'impact économique de la culture et des communications dépasse celui de l'agriculture, des mines, des pêcheries et du tourisme ${ }^{18}$. N'est-ce pas une raison pour en faire un actif privilégié du développement et pour l'affirmer?

\section{Un questionnement}

Je formule ici quelques questions.

\section{Un repositionnement de la culture}

Cette politique expose un repositionnement de la culture sur l'échiquier gouvernemental. Pourquoi ne pas y avoir ajouté le binôme Culture et immigration, le débat de la laïcité qui sous-tend une posture interculturelle se faisant à nouveau jour, ainsi que celui de Culture et développement durable, lequel trouvait déjà une place dans l'Agenda 21 de la culture ? Et pourquoi ne pas imaginer un binôme Culture et régions?

\section{Une journée gratuite par mois dans les musées}

Cet objectif est inscrit sous la rubrique de faciliter l'accès aux musées. On peut

17. Georges-Émile Lapalme, Pour une politique, le programme de la révolution tranquille, Montréal, VLB Éditeur et Maria Lapalme, «Études québécoises », 1988, p. 77.

18. Le secteur primaire de l'économie représentait en 2014 2,8\% du produit intérieur brut (Рів) mis à jour en 2018 alors que celui de la culture représentait $12,3 \mathrm{G} \$$, soit 3,2\% du même PIB et il générait 191500 emplois. Source : « Le Québec économique », portail d'information sur l'économie du Québec, à partir de données de Statistique Canada, 2014. 
déjà présumer que le nombre de visites augmentera, mais s'agira-t-il de celles de nouveaux citoyens qui viendront découvrir le musée ou plutôt de celles des habitués qui profiteront d'une telle journée gratuite? Les initiatives de la France sous ce rapport laissent planer un doute : « La construction frénétique de musées et de théâtres en trente ans a provoqué une forte augmentation mais ce sont les aficionados qui y vont plusieurs fois tandis que les ouvriers et jeunes de banlieue y vont moins... Le fossé se creuse. ${ }^{19}{ }^{\prime}$ C'est désormais connu que les politiques de l'offre n'élargissent plus le bassin de publics et qu'il est plus utile de prendre appui sur la dimension sociale des arts et du patrimoine comme le propose la politique dans le même chapitre.

\section{Les bibliothèques}

N'oublions pas que les bibliothèques sont un puissant maillon d'accès à la culture dont l'avenir peut s'avérer fragile. Cette politique propose déjà, on l'a vu, un accompagnement en matière de culture dans les bibliothèques municipales pour les personnes immigrantes et nouvellement arrivées au Québec. Certaines d'entre elles jouent déjà un rôle actif dans le soutien aux devoirs des enfants afin de favoriser la maturité scolaire. Mais pourquoi ne pas suivre l'exemple de la ville de Toronto, qui s'inscrit dans les villes intelligentes, lesquelles accumuleront des quantités considérables d'informations sur les citoyens, et qui choisit de miser sur la longue et grande expertise des bibliothèques qui ont su maîtriser l'entreposage de l'information plutôt que de créer un organisme de gestion de ces données ou de les confier au privé ${ }^{20}$ ? N'y a-t-il pas ici un nouveau partenariat culturel et de communication à explorer entre le gouvernement et les villes?

\section{Le droit d'auteur}

Nous sommes confrontés aujourd'hui à un grave problème universel de droits d'auteurs. Cette responsabilité est celle du gouvernement du Canada et il a entrepris une révision de cette loi. La marge de manœuvre du gouvernement québécois est bien mince sous ce rapport, mais il doit jouer son rôle de portevoix des créateurs.

\section{Musées et municipalités}

Les institutions muséales disposent d'expertises riches et variées : collectionnement, archives, mise en valeur d'œuvres et d'objets, mise en scène, stratégies de communications avec les publics. Ne pourrait-on pas ici encore ouvrir un autre axe de partenariat avec les municipalités afin de soutenir des

19. Olivier Donnat, « Le Sociologue qui casse le moral», Le Monde, 26 octobre 2018.

20. Maxime Johnson, « Les Bibliothèques au cœur de la ville intelligente ? », L'Actualité, mai 2019, p. 11. 
initiatives d'interprétation historique dans les parcs, $d$ 'aménagement de places publiques par des œuvres d'art, renforçant alors l'insertion du musée dans la collectivité et favorisant une concertation de ressources avec ces mêmes municipalités.

\section{Le financement}

Voilà la question névralgique de toute politique. Les deux gouvernements successifs ont pris des engagements de plus de 500 millions de dollars pour l'un auquel l'actuel gouvernement a décidé d'ajouter à son tour 300 millions au cours des cinq prochaines années. Nous n'avons jamais vu de pareilles sommes offertes à la culture et aux communications. On a même procédé à une ventilation par engagement pour rendre le propos encore plus réel, bien que les sommes qui pourraient survenir pour soutenir les médias n'y fussent que partiellement comptabilisées.

Deux questions intriguent. Ainsi, lorsque l'on compare les dépenses de l'année 2018-2019 et les engagements pris pour l'exercice financier en cours de 2019-2020, l'écart est très faible, car la majoration n'est pas inscrite au Livre des crédits du gouvernement pour la Culture et les communications, mais plutôt annoncée comme devant être puisée au fonds de suppléance. De même, les crédits prévus pour soutenir les médias sont annoncés comme devant être « pourvus à même les disponibilités budgétaires dégagées en cours d'exercice. ${ }^{21} \gg$ De tels crédits budgétaires seront-ils pérennes pour la suite ?

En second lieu, il est très difficile d'établir quelle est la proportion des crédits de cette politique qui seront inscrits dans les dépenses d'autres ministères. Ce que le Livre des crédits permet de voir, c'est qu'une somme d'environ 591 millions de dollars émargerait au budget de la culture au cours des cinq prochaines années. Faut-il présumer que le solde devrait être réparti entre les autres ministères ou organismes gouvernementaux, soit environ 209 millions ?

\section{Conclusion}

Le Québec occupe une place enviable sur le plan international, car il a su obtenir, par la qualité de ses artistes et de ses organisations, une reconnaissance de la qualité de sa création. Cette politique tient d'importantes promesses. Mais quel est son espace culturel sur le Web ? Le numérique qui change la manière de communiquer change également la manière d'exister socialement et collectivement. Cette politique culturelle tente, par certaines dispositions, de baliser Internet et ses effets sur les arts, les lettres, les industries culturelles et le patrimoine. Toutefois ces nouvelles avenues de communication et d'information ont un effet beaucoup plus considérable et transforment la culture des sociétés. Il est au demeurant symptomatique que cette politique

21. Conseil du Trésor, Livre des crédits 2019-2020, section Culture, p. D 67 à D 73. 
évoque rarement la notion identitaire qui a porté les politiques culturelles dans la seconde moitié du $\mathrm{Xx}^{\mathrm{e}}$ siècle.

Comment parvenir à ce que la place des arts et des lettres conserve un tant soit peu des traces de ses origines issues de la Renaissance et de l'ÉtatNation apparu avec le traité de Westphalie en $1648^{22}$ ? Comment positionner les cultures nationales qui sont le socle de la diversité des sociétés sur la planète dans un monde en perpétuelle mouvance et sollicité par des gestes interculturels? Tel est le décor dans lequel se profilent les orientations de cette politique culturelle que l'État veut soutenir. Il y a peu encore, nous avions l'espoir que la culture et les arts contribuent à former des citoyens de demain qui enrichissent à leur tour les sociétés. Ce rôle serait-il en train de basculer, tellement les tendances contemporaines semblent à l'opposé de la pensée de l'UNESCO de la seconde moitié du $\mathrm{Xx}^{\mathrm{e}}$ siècle ? La rapidité des changements décale déjà des segments de cette politique. Lorsque l'intelligence artificielle prendra bientôt place dans le jeu des algorithmes qui déterminent les critères de visibilité des œuvres, qu'arrivera-t-il ? Une politique n'a pas pour objectif de prévoir l'avenir, mais elle a la responsabilité de se doter de moyens pour lire le changement et s'y adapter. Imaginons au terme des cinq premières années d'implantation ce que cela pourrait signifier. Le Ministère ne devrait-il pas se donner comme passage obligé des étapes d'évaluation périodique pour ne pas s'en trouver décalé à son tour?

Comment ces changements affecteront-ils la culture dans les accords internationaux, dans la capacité de petites sociétés de se tailler une place au-delà de l'événementiel à court terme sur l'échiquier international ? Une politique culturelle réussie a les vertus de voir ses interlocuteurs surtout satisfaits des choix et des engagements proposés, mais elle s'inscrit également dans des choix de sociétés. Ce me semble être le cas présentement, bien qu'un certain nombre de points demeurent toujours obscurs.

Finalement, permettez-moi de paraphraser l'historien Pierre Nora qui affirmait récemment au quotidien Le Monde que, dans un contexte de grandes incertitudes, " nous savons qu'il y a un futur, mais il se pourrait que nous n'ayons plus d'avenir ${ }^{23} »$. Alors, « [s]e pourrait-il [...] que nos choix culturels soient ce mortier puissant entre les pierres pour nous donner des accents de solidarité intérieure et internationale, de cohésion sociale et de tolérance culturelle... ${ }^{24} \gg$ qui pourraient atténuer les secousses « des incertitudes soulevées par les récentes mutations de nos sociétés ${ }^{25} »$ ?

22. Francine Pelletier, « Cent ans d'incertitude », Le Devoir, 13 février 2019.

23. Pierre Nora, «2019. La fin d'un monde n'est pas la fin du monde », Le Monde, 21 mars 2019.

24. Gérald Grandmont, La Culture, un capital à faire fructifier, Montréal, Chaire en gestion des arts Carmelle-et-Rémi-Marcoux, HÉc-Montréal, 2016, p. 168.

25. Ibid.; Nora, op. cit. 